\title{
How Does Collagen Adsorb on Hydroxyapatite? Insights From Ab Initio Simulations on a Polyproline Type II Model
}

\author{
Michele Cutini, ${ }^{\dagger}$ Marta Corno, ${ }^{\dagger, \S(0)}$ Dominique Costa, ${ }^{\ddagger}{ }^{\circ}$ and Piero Ugliengo ${ }^{*}, \dagger$ (1) \\ ${ }^{\dagger}$ Department of Chemistry and NIS (Nanostructured Interfaces and Surfaces) Center, University of Turin, Via P. Giuria 7, 10125 \\ Turin, Italy \\ ${ }^{\ddagger}$ Chimie ParisTech, PSL University, CNRS, Institut de Recherche de Chimie Paris, Physico-Chimie des Surfaces, 11 rue P et M Curie, \\ 75005 Paris, France
}

\section{Supporting Information}

\begin{abstract}
Bone has a hierarchical structure based on the mineralized fibril, an organic matrix envisaging collagen protein in tight interaction with hydroxyapatite mineral (HAP) and stabilized by water molecules. The tremendous complexity of this natural composite material hides the extraordinary features in terms of high compressive strength and elasticity imparted by the collagen protein. Clearly, understanding the nanoscale interface and mechanics of bone at atomistic level can dramatically improve the development of biocomposite and the understanding of bone related diseases. In this work, we aim at elucidating the features of the interaction between a model of a single-collagen-strand (COL) with the most common dried P-rich (010) HAP surface. The methods of choice are static and

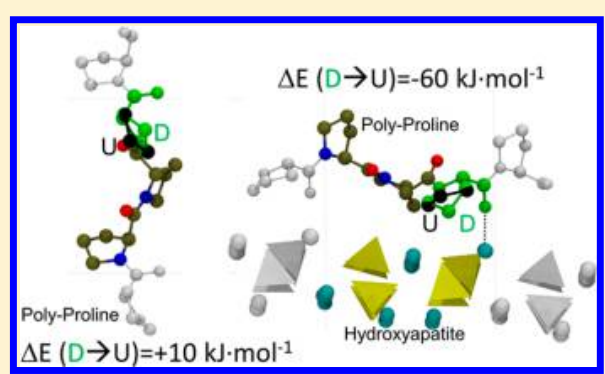
dynamic simulations based on density functional theory at PBE-D2, PBE-D3 and B3LYP-D3 levels. Collagen is made to a large extent by proline (PRO) and derivatives, and PRO's side chain is known to affect the collagen triple helix stability dramatically. However, the role of the PRO side chain in the COL/HAP interface has never been studied so far at a quantum mechanical level. To decrease the enormous structural complexity of collagen itself, we employed a simple collagen model, i.e., a single strand based on the poly-L-proline type II polymer (PPII), which, for its composition, nicely suites our purposes. We discovered that during the HAP adsorption process, the polymer deforms to create a relatively strong electrostatic interaction between the PRO carbonyl $\mathrm{C}=\mathrm{O}$ group and the most exposed $\mathrm{Ca}$ ion of the P-rich (010) HAP surface. Both dynamic and static simulations agree that the HAP surface guides the formation of PPII conformers, which would be unstable without the support of the HAP surface. The PROs puckering and the polymer orientation affect the PPII affinity for the HAP surface with binding energies spanning within the $63-126 \mathrm{~kJ} \cdot \mathrm{mol}^{-1}$ range. This work is the first step toward the development of a full collagen model envisaging a threeinterlocked helical polymer interacting with the HAP surfaces.
\end{abstract}

\section{INTRODUCTION}

Bone is a fundamental biomaterial with intriguing mechanical properties. It has a hierarchical structure based on the mineralized fibril, which is an organic matrix stabilized by water, macro-molecules, and a mineral reinforcement. ${ }^{1,2}$ The organic and inorganic parts in bone are the collagen protein and the hydroxyapatite mineral (HAP), respectively. ${ }^{2}$ The collagen protein is a triple polypeptide helix $1.25 \mathrm{~nm}$ wide and $300 \mathrm{~nm}$ long, defined by a central triple helical domain with two short non helical regions at the ends, called telopeptides. The central helical domain is composed by three parallel polypeptides strands wrapped together, where each strand has a poly proline type II (PPII) geometry. ${ }^{1}$ The primary structure of collagen helical domain is restricted to a triplet repeat sequence, where Glycine (GLY) is always in first position. Proline (PRO) and Hydroxy-Proline (HYP) are the most common amino acids in the second and the third position. In all collagens, the GLYPRO-HYP (GPH) triplet is the most common triplet (10.5\%). ${ }^{3}$ From a structural point of view, GLY is buried inside the core of the protein, and the second and third amino acids in the triplet are usually the most exposed. The three peptide strands are kept together by $\mathrm{H}$-bonds between the carboxylic group of the amino acid in the second position and the $\mathrm{N}-\mathrm{H}$ of GLY. ${ }^{4}$ In bone, groups of five collagen helices organize into microfibrils liked together by the sharing of one them. ${ }^{5}$ Many microfibrils packed together create a fibril with diameter ranges around 100-200 nm. The overall fibril is well ordered and exhibits a $67 \mathrm{~nm}$ periodicity along the protein axis, where a hole zone between the ends of the collagens (40 nm long) is followed by an overlap zone ( $27 \mathrm{~nm}$ long). ${ }^{6}$ In the holes and outside the fibril, HAP mineralizes in the form of flat nanoplatelets $\left(3-4 \mathrm{~nm}\right.$ thick). ${ }^{7,8}$ It is still not clear whether the HAP is in major amount inside or outside the fibril, ${ }^{2}$ but in all cases the mineral grows mainly along the $c$ crystallographic axis, exposing the $\{010\}$ surface. ${ }^{9}$ The $c$ axes (to which the $\mathrm{OH}$

Special Issue: Hans-Joachim Freund and Joachim Sauer Festschrift

Received: October 10, 2017

Revised: December 13, 2017

Published: December 18, 2017 
channel is aligned) of all HAP nanoplatelets lie parallel to each other and also to the collagen protein axis. ${ }^{2,10}$

Many theoretical works investigating the bone material have been carried out in the past decade. Most of them have been focused on its mechanical properties, ${ }^{11,12}$ without providing much details of the organic/inorganic interface geometry and energetic. Due to the extraordinary complexity of this composite material, modeling studies almost rely on ad hoc force fields allowing performance of classical molecular dynamic calculations (MD). ${ }^{12-16}$ Even though the MD approach allows one to treat a much higher number of atoms per simulation than quantum mechanical (QM) methods, ${ }^{17}$ massive parallel codes coupled with high performance computing (HPC) resources have pushed forward the limit size also for $\mathrm{QM}$ methods. A recent successful example is the static investigation of a protein crystal within the hybrid density functional theory approach. ${ }^{18,19}$ True AIMD is clearly also feasible with highly specialized and efficient computer codes. ${ }^{20}$

In our research group, we started investigating the HAP surface/biomolecule interaction past decade, by employing a bottom-up approach based on DFT model chemistry. ${ }^{21}$ We have elucidated the adsorption of water molecules, ${ }^{22}$ simple amino-acids $^{23-25}$ up to peptides ${ }^{26}$ on different HAP surfaces through a robust computational approach, often compared successfully with experiments. ${ }^{21}$ In this work, we focused on the collagen-single-strand/HAP interaction to model the organic/ inorganic interface in bone. We adopted the $\{010\}$ P-rich HAP slab (2D periodic) as the HAP nanoplatelet model, and the poly proline type II polymer (1D periodic), PPII, as a model of a single strand of collagen. PPII is a good collagen model due to the high content of imino-acids in collagens and the geometry of each single strand. Both HAP surface and polymer models have been investigated in details in previous works. ${ }^{21,27}$ The use of a polymeric system as a collagen model allows us to get rid of the $\mathrm{COOH}-\mathrm{NH}_{2} /$ surface interaction, which is usually the leading force in the adsorption process of small molecular peptides onto surfaces blurring the real interaction between the core peptide and the mineral surface atoms. ${ }^{17}$ This is even more so because of the very long collagen axial length: therefore, simulating the collagen with short peptide, i.e. molecular PRO tripeptide, will emphasize the role of terminal groups, with misleading results. We aligned the polymer along the $b$ HAP vector. Even though in bones the experimental collagen alignment is along the a HAP vector (parallel to the $\mathrm{O}-\mathrm{H}$ channel), recent AFM and MD simulations indicated that the preferred one has an inclination of $72.5^{\circ}$ with respect to the $a$ vector on the $\{010\}$ face of HAP. Although the PPII main chain has only one available conformation imposed by the polymeric nature of the model, the side chain flexibility allows the coexistence of four PPII conformers (see the Molecular Models section for further details). It is well-known that the role of PRO side chain conformations in the collagen triple helix stability is enormous. ${ }^{4,28}$ Therefore, our aim in this work is to unravel its effect on the adsorption process of PPII on HAP. We computed the optimized adsorption geometries by means of static DFT simulations inclusive of the dispersion corrected (D2) PBE functional. The adsorption of all side chain conformers and two polymer alignments along the $b$ HAP vector have been investigated. We calculated the energy of the process with both PBE and B3LYP functionals coupled with the $\mathrm{D} 2$ and $\mathrm{D} 3^{\mathrm{ABC}}$ dispersion schemes. Moreover, to include thermal and entropic contributions in our simulations, we investigated the dynamic evolution of the polymer as free and in interaction with the HAP surface by means of AIMD simulation at PBE-D2 level. Both static and dynamic calculations demonstrate that the surface has a fundamental role in guiding the formation of specific PPII conformers. The results of this work are the basis for further investigations, currently on going in our laboratory, on a more realistic collagen model based on a triple helix interacting with the HAP surface.

\section{COMPUTATIONAL METHODS}

All the calculations relied on the DFT theory and were performed with a development version of the CRYSTAL14 code. ${ }^{29,30}$ Relaxed geometries were calculated employing the pure GGA PBE functional, coupled with the D2 dispersion scheme, to account for dispersion forces. ${ }^{31,32}$ For all the simulations, the $\mathrm{D} 2$ dispersion energy was scaled by a factor of 0.75 , and the $\mathrm{C}_{6}$ coefficient of the $\mathrm{Ca}$ atom is set to zero, considering that the original $\mathrm{C}_{6}$ values were derived for neutral isolated atoms while, in the HAP case, $\mathrm{Ca}$ atoms are almost double-charged ions with a dramatic reduction of their polarizability. In the geometry relaxation of the PPII/HAP adduct, the cell vectors were kept fixed to the optimum values of the free HAP slab. Atomic positions and cell vectors optimization adopted the analytical gradient method. The Hessian was upgraded with the Broyden-Fletcher-GoldfarbShanno (BFGS) algorithm. ${ }^{33-35}$ We set tolerances for the convergence of the maximum allowed gradient and the maximum atomic displacement to the CRYSTAL default values (0.00045 Ha.bohr ${ }^{-1}$ and 0.0018 Bohr, respectively). The $\Gamma$ centered k-point grid was generated via the Monkhorst-Pack scheme ${ }^{36}$ with a system-dependent number of $\mathrm{k}$ points. For the geometry optimizations, the shrinking factor is set to 11 and 4 4 for the PPII/HAP and HAP cases. For the free PPII calculations, the shrinking factor is set to 4 . These choices ensured good convergence on the number of $\mathrm{k}$ points for the energy and geometry of the models employed. To help convergence of the SCF, the Fock/KS matrix at a given cycle was mixed with $30 \%$ of the one of the previous cycle. ${ }^{37}$ The electron density and its gradient were integrated over a pruned grid consisting of 974 angular and 75 radial points generated through the Gauss-Legendre quadrature and Lebedev schemes. ${ }^{38}$ The recently introduced DIIS extrapolator technique, which reduces the number of SCF cycles by almost a factor 2, has been employed to speed up the SCF convergence. ${ }^{39}$ Tolerance values controlling the Coulomb and exchange series (the latter for B3LYP calculations) in periodic systems ${ }^{37}$ are set to $10^{-6}$ for Coulomb overlap, Coulomb penetration, exchange overlap and exchange pseudo-overlap in the direct space, and $10^{-14}$ for exchange pseudo overlap in the reciprocal space. Valence triple- $\zeta$ polarized (VTZP) basis sets were adopted for carbon, nitrogen, and oxygen atoms, ${ }^{40}$ while hydrogen, calcium and phosphorus atoms are described with a 3-1G(p), $865-1 G(p, d)$, and $85-2 G(p, d)$ basis set, respectively. ${ }^{41}$

It is worth noting that, at variance with codes adopting plane waves basis set, the HAP surfaces computed within the CRYSTAL14 "slab model approach" are represented as true 2D systems (no vacuum needed because no slab replica exists), thanks to the adoption of Gaussian type orbitals. The same applies when treating the isolated PPII, which is represented as a true isolated $1 \mathrm{D}$ polymer.

Binding energies (BEs; see next paragraph) were estimated with both the PBE and the B3LYP hybrid functionals. ${ }^{42-44}$ Hybrid derived energy data were computed by single point 
energy evaluation at B3LYP-D3 ${ }^{\mathrm{ABC}}$ on the PBE-D2 optimized structures, hereafter referred to as B3LYP-D3 ${ }^{\mathrm{ABC}} / / \mathrm{PBE}-\mathrm{D} 2$ notation. The long-range weak forces were estimated through both the D2 (see ref 32) and the most recent $\mathrm{D} 3{ }^{\mathrm{ABC}}$ dispersion schemes (see Table S5). ${ }^{45-47}$ In order to increase the accuracy of the computed $\mathrm{BE}$, single energy point evaluations were computed with shrinking factor set to 44 .

$\mathrm{Ab}$ initio molecular dynamics (AIMD) simulations were performed using the $\mathrm{CP} 2 \mathrm{~K}$ code. ${ }^{48}$ The Quickstep technique with a mixed plane wave and Gaussian basis set methodology (Gaussian and Plane Wave method, GPW) was employed to calculate the electronic structure. ${ }^{49}$ We used the PBE functional, with the Goedecker-Teter-Hutter pseudopotentials, ${ }^{50}$ and a Gaussian triple- $\zeta$ basis set with polarization functions (TZVP) for all atoms, but $\mathrm{P}$ and $\mathrm{Ca}$ for which a double- $\zeta$ basis set with polarization functions (DZVP) is employed. The cutoff for the plane wave basis was set to 400 Ry. Dispersion forces are taken into account with the Grimme D2 method. For the PPII system, AIMD simulations were run at $300 \mathrm{~K}$ in the isothermal isobaric ensemble (NPT) at zero external pressure (temperature of the barostat is set to $50 \mathrm{~K}$ ) using a cell, flexible only along the polymer axis. Tolerances on the temperature fluctuation is set to $\pm 50 \mathrm{~K}$. A reference cell was set to avoid unphysical jumps in energy. The simulation for the PPII system is run for 22.6 ps. For the PPII/HAP system, AIMD dynamics were conducted in the microcanonical NVE ensemble (after equilibration is achieved through canonical NVT dynamics at $T=300 \mathrm{~K}$ ) with a time-step of $0.5 \mathrm{fs}$. For the free PPII polymer, the cell parameters, that are perpendicular to the polymer axis, are fixed to $26 \AA$, introducing vacuum space for separating polymer images. For the PPII/HAP system, we fixed the $a$ and $b$ cell parameters to the PBE-D2 CRYSTAL optimum values, separating $2 \mathrm{D}$ images by a $c$ axis of $36.2 \AA$. The equilibration time for the PPII/HAP system is $2404.5 \mathrm{fs}$, and the production time is $10 \mathrm{ps}$. In all cases, a time step of 0.5 fs was chosen, and the Nosé-Hoover thermostat was employed for both NPT and NVT dynamics. ${ }^{51,52}$

The graphical visualization and structural manipulation of structures was performed with MOLDRAW version 2.0. ${ }^{53}$ Dynamical data analysis was performed employing the VMD program. ${ }^{54}$ Images were rendered with VDM and VESTA software. $^{55}$

Interaction Energies. In a periodic treatment, the binding energy (BE) per unit cell per adsorbate is defined as

$$
\mathrm{BE}=E(\mathrm{~S} / / \mathrm{S})+E_{\mathrm{P}}(\mathrm{P} / / \mathrm{P})-E(\mathrm{SP} / / \mathrm{SP})
$$

where the symbol following the double slash identifies the optimized geometry at which the energy has been computed. Therefore, $E(\mathrm{~S} / / \mathrm{S}), E_{\mathrm{P}}(\mathrm{P} / / \mathrm{P})$, and $E(\mathrm{SP} / / \mathrm{SP})$ are the energies of a bare HAP slab $S$, the free PPII polymer $\mathrm{P}$ and the interacting PPII/HAP system SP in their fully optimized geometry. BE is a positive quantity for a bounded system and can be recast in terms of the geometrical deformation cost of the surface $\left(\delta E_{\mathrm{S}}>0\right)$, the adsorbate $\left(\delta E_{\mathrm{P}}>0\right)$, the lateral adsorbate-adsorbate interactions $\left(\Delta E_{\mathrm{L}}\right)$ between polymer images, and the binding energy between the predeformed constituents $\left(\mathrm{BE}^{*}\right)$ :

$$
\begin{aligned}
& \delta E_{\mathrm{S}}=E(\mathrm{~S} / / \mathrm{SP})-E(\mathrm{~S} / / \mathrm{S}) \\
& \delta E_{\mathrm{P}}=E_{\mathrm{P}}(\mathrm{P} / / \mathrm{SP})-E_{\mathrm{P}}(\mathrm{P} / / \mathrm{P}) \\
& \Delta E_{\mathrm{L}}=E(\mathrm{P} / / \mathrm{SP})-E_{\mathrm{P}}(\mathrm{P} / / \mathrm{SP})
\end{aligned}
$$

$$
\begin{aligned}
& \mathrm{BE}^{*}=E(\mathrm{~S} / / \mathrm{SP})+E(\mathrm{P} / / \mathrm{SP})-E(\mathrm{SP} / / \mathrm{SP}) \\
& \mathrm{BE}=\mathrm{BE}^{*}-\delta E_{\mathrm{S}}-\delta E_{\mathrm{P}}-\Delta E_{\mathrm{L}}
\end{aligned}
$$

$\Delta E_{\mathrm{L}}$ is the difference between the energy of the PPII chain organized as a periodic 2D system in the SP geometry and the energy of a single deformed PPII polymer. This term can then be either positive/negative as a function of the favorable/ unfavorable PPII interchain interaction energy. Obviously, BE* $>\mathrm{BE}$, as the deformation costs are usually larger than the lateral interactions $\Delta E_{\mathrm{L}}$, even when the latter are repulsive. As we are dealing with localized Gaussian-type basis set, we should correct the BE for the basis set superposition error (BSSE) $)^{56}$ using a well consolidated procedure already adopted in previous works. ${ }^{22}$ Only $\Delta E_{\mathrm{L}}, \mathrm{BE}^{*}$, and $\mathrm{BE}$ terms are affected by BSSE (all deformation terms are BSSE free), and their corrected values are indicated with the notation $\Delta E_{\mathrm{L}}{ }^{\mathrm{C}}, \mathrm{BE}{ }^{* \mathrm{C}}$, and $\mathrm{BE}^{\mathrm{C}}$, respectively. To avoid a relevant role of the lateral interactions between polymer replicas, which would have obscured the importance of the HAP surface, we adopted a unit cell size large enough to ensure that the value of $\Delta E_{\mathrm{L}}$ was negligible. For our purposes (see Figure 6), we need an alternative definition for the deformation energy of the polymer.

$$
\Delta E_{\mathrm{P}}=E_{\mathrm{P}}(\mathrm{P} / / \mathrm{SP})-E_{\mathrm{P}}(\mathrm{P} / / \mathrm{P})^{*}
$$

where $E_{\mathrm{P}}(\mathrm{P} / / \mathrm{P}) *$ is the energy of the polymer with cell length fixed to $9.538 \AA$. It worth noting that $\Delta E_{\mathrm{P}}$ and $\delta E_{\mathrm{P}}$ differ only for the energy cost of the polymer unit cell stretching.

\section{RESULTS AND DISCUSSION}

Molecular Models. To model the HAP surface, we considered the nonstoichiometric (P-rich) (010) surface, modeled through the slab approach as cut out from the HAP bulk optimized structure. This surface was shown by some of $\mathrm{us}^{23}$ to be a key surface in adsorption of amino acids on HAP nanocrystals. Further details on the relevance of the different HAP surfaces can be found in a recent review paper. ${ }^{21}$ The slab unit cell ( $2 \mathrm{D}$ periodic) adopted in ref 23 contained 114 atom and was $20 \AA$ thick (see Figure 1). This slab envisages alternating electro-neutral layers of $\mathrm{Ca}_{3}\left(\mathrm{PO}_{4}\right)_{2}$ (A-type) and $\mathrm{Ca}_{4}\left(\mathrm{PO}_{4}\right)_{2}(\mathrm{OH})_{2}$ (B-type), as shown in Figure 1. Here, for saving computational resources, we reduced the slab thickness to the $\mathrm{A}-\mathrm{A}-\mathrm{B}-\mathrm{A}-\mathrm{A}$ layers only (see Figure 1 ), resulting in 70 atoms in the unit cell and a thickness of $12 \AA$. We carefully

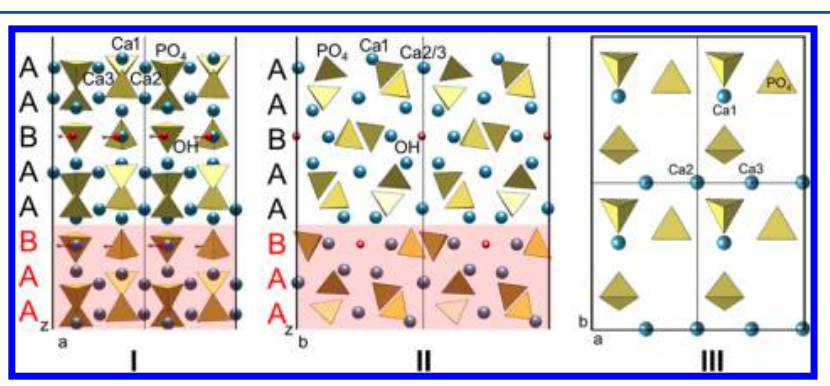

Figure 1. Views of the HAP (010) slab model as defined in ref 23 along the [100], [010], and [001] directions (I, II, and III, respectively). The unit cell slab adopted here only includes the A-AB-A-A layer sequence, while layers in the reddish area (A-A-B) are removed. The $\mathrm{OH}$ containing channel runs parallel to the $b$ axis. Color coding: $\mathrm{PO}_{4}$ group (yellow tetrahedron), calcium (cyan spheres), oxygen (red spheres), and hydrogen (light gray spheres). 
checked that the slab reduction did not alter the interatomic distances and Mulliken charges compared with the values computed for the thicker slab. Internal coordinates and cell parameters of the HAP model were fully relaxed at the PBE-D2 level of theory. The resulting relaxed cell parameters are $a=$ $6.993, b=9.538$, and $\gamma=90.02$. In the PPII/HAP study we employed a HAP supercell doubling the size along the $a$ axis ( $a$ $=13.986, b=9.538, \gamma=90.02$, and 140 atoms $/$ cell) to reduce the lateral interaction between PPII chain by bringing the $\Delta E_{\mathrm{L}}$ term close to zero (vide supra).

The collagen protein model is a polyproline type II polymer (PPII) with a unit cell containing three PROs. Each PRO has two stable side chain conformations, labeled DOWN and UP. A PRO is defined as DOWN (D) or UP (U) for positive or negative values of the $\chi^{2}$ dihedral angle (see Figure 2), which

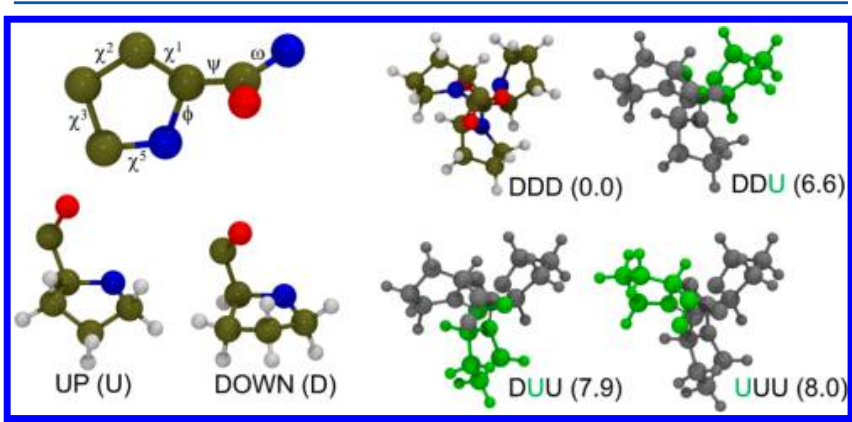

Figure 2. Top left: definition of the torsional angles of a PRO belonging to the PPII polymer. Bottom left: UP (U) and DOWN (D) PRO puckering. Right: Views along the polymer main axis of the PPII conformers. The relative energy $\left(\mathrm{kJ} \mathrm{mol}^{-1}\right)$ of the conformers with respect to the most stable DDD conformer is given in parentheses. Color coding for atoms: carbon (green), nitrogen (blue), oxygen (red), and hydrogen (light gray).

controls the ring puckering. In theory, the PRO is $\mathrm{D} / \mathrm{U}$ when the ring is in an envelope conformation with $\mathrm{C} \gamma$ out of plane in the same/opposite side of the $\mathrm{C}=\mathrm{O}$ group (see Figure 2). Therefore, four stable PPII conformers can be defined, i.e., DDD, UDD, UUD, and UUU. Among them, the DDD is the most stable one (Figure 2). The characteristics and the interconversion between these conformers have been deeply investigated in a previous work by some of us. ${ }^{27}$ The internal coordinates of each PPII conformer have been relaxed by forcing the unit cell length fix to $9.538 \AA$ to allow exact matching between the HAP slab and PPII polymer unit cell. It is relevant to compare this value with the shorter one of 9.120 A resulting from the full optimization of the free PPII polymer (see Table S1). In the following, we will also study the effect that the shrinking of the unit cell brings about on the conformational energy of the PPII polymer.

Polymer Docking. The HAP (010) P-rich surface exposes $\mathrm{Ca}$ cations embedded in a matrix of $\mathrm{PO}_{4}$ anions, while $\mathrm{OH}$ anions are hidden within the slab and play no role in adsorption. The electrostatic potential rendered over the surface of constant electron density (see Figure 3, right) shows well-defined regions of relatively high positive/negative values, which are active in the adsorption of biomolecules. The corresponding electrostatic potential of the PPII (Figure 3, left) reveals only important deviation from neutrality in the proximity of the $\mathrm{C}=\mathrm{O}$ groups, in which negative (red colored) basins of potential occur. We have therefore docked the PPII polymer toward the HAP (010) P-rich surface following the

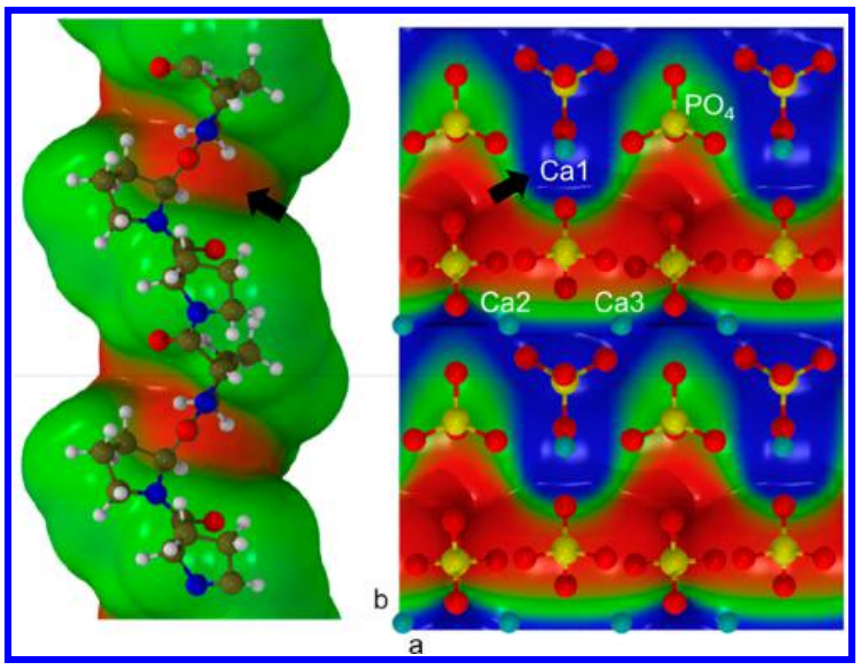

Figure 3. Left: PBE PPII polymer electrostatic potential surface viewed perpendicularly to the main PPII axis. Right: top view along the $z$ axis (as in Figure 1,III) of the PBE electrostatic potential surface of the P-rich (010) HAP surface. For both cases, the electrostatic potential surface is mapped on the electron density envelope of value 5 $\times 10^{-5}$ a.u. Positive/negative value of the electrostatic potential are reported as blue/red colors $( \pm 0.02 \mathrm{au})$. Color coding for atoms: carbon (green), nitrogen (blue), calcium (cyan), phosphorus (yellow), oxygen (red), and hydrogen (light gray).

electrostatic complementarity principle, i.e., by maximizing the match between the blue/red zones of the respective maps. Considering that the Cal is the most active (and exposed; see Figure 1, top-left) Ca ion on the surface, we oriented the PPII $\mathrm{C}=\mathrm{O}$ group toward it. The alignment of the PPII polymer with the HAP $b$ vector can occur in two different orientations, named hereafter D1 and D2, as shown in Figure 4. D2 is related to $\mathrm{D} 1$ by a rotation of $180^{\circ}$ around the $z$-axis perpendicular to the HAP surface. In both cases, the PRO1 carboxylic group points toward the Cal surface atom with the PRO1 pyrrolidine ring lying parallel to the HAP surface. In PRO2 and PRO3 residues, the $\mathrm{C}=\mathrm{O}$ group points away from the surface with the pyrrolidine ring lying parallel and normal to the HAP surface, respectively.

To fully explore the role of the PRO side chain in the PPII/ HAP adduct, we investigated the interaction with the surface of all PPII conformers, e.g., DDD, UDD, UUD, and UUU. For UDD and UUD, there are three different ways to place the PPII polymer at the surface, as a function of the position of the triplet sequence. In the UDD/UUD cases, the U/D puckered PRO may occur in PRO1, PRO2, or PRO3 amino acids (see Figure 4). We labeled all possible cases as UDD/HAP-DUU/ HAP, DUD/HAP-UDU/HAP and DDU/HAP-UUD/HAP, respectively. The puckering of the PRO in PRO1 position is always reported in italic. To avoid confusion between the conformers of the free and adsorbed polymer, from now on, we will refer to the latter case following the format $\mathrm{XXX} / \mathrm{HAP}$, with $\mathrm{X}=\mathrm{D}$ or $\mathrm{U}$.

Geometrical and Energetic Features from Static Calculations. In this section, we report on the adsorption of the PPII polymer to the HAP surface by means of static PBED2 simulations. Starting with the D1/D2 alignments of the polymer (see Figure 4), all resulting PPII conformers have been characterized in the adsorption state at the HAP surface. The docking geometry is characterized by the PRO1 carboxylic group pointing to the HAP Cal atom (see the Molecular 


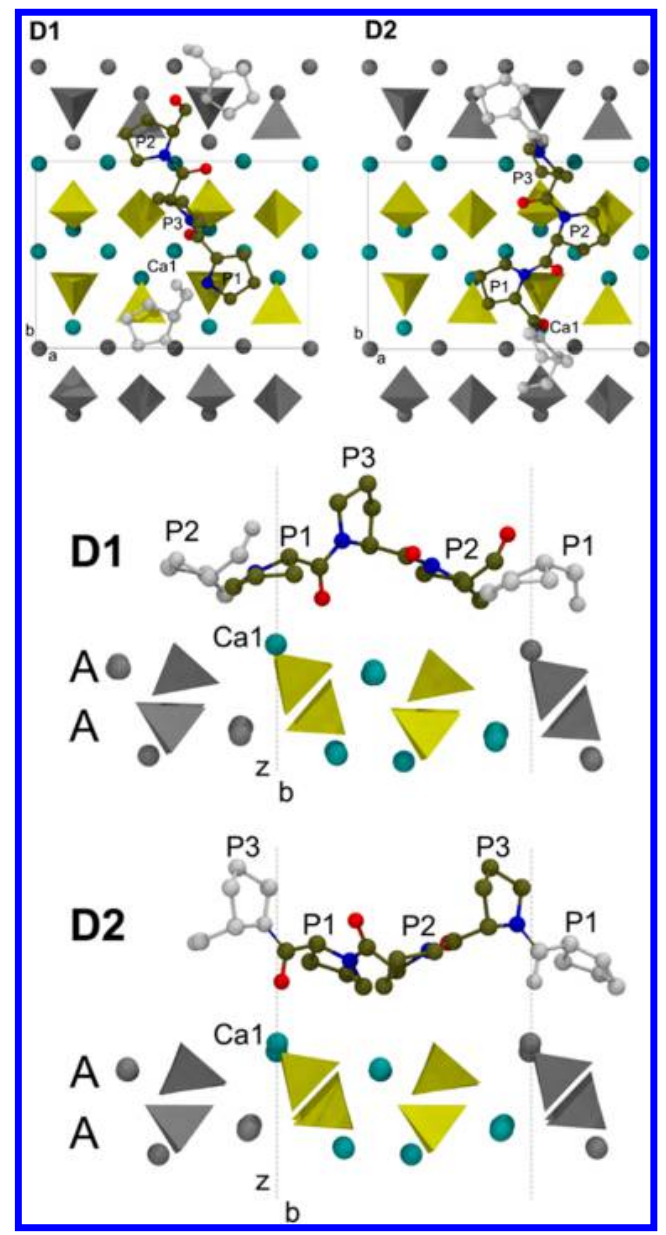

Figure 4. Top: top view along the $z$-axis of the adsorbed UDD PPII polymer oriented in the D1 and D2 ways. Bottom: lateral view of the UDD PPII polymer adsorbed on (010) P-rich HAP surface in the D1 and D2 alignments. Only the first two layers (AA) of HAP are reported, for the sake of clarity. Color coding for atoms inside the unit cell: carbon (green), nitrogen (blue), calcium (cyan), $\mathrm{PO}_{4}$ (yellow tetrahedron), oxygen (red). The atoms outside the unit cell are in white (PROs) and silver (HAP). $\mathrm{H}$ atoms are hidden for the sake of clarity.

Models section for further details). Computed binding energies (BE) and geometries of adsorption are reported in Tables 1 and 2 .

Regardless of the PROs puckering and the D1/D2 alignment, PPII adsorption on HAP is a favorable process with BE values in the 63-126 $\mathrm{kJ} \cdot \mathrm{mol}^{-1}$ range (see Table 1), with D1 envisaging the most stable systems (UDD and UUD). The geometry optimization procedure confirms a specific $\mathrm{C}=\mathrm{O} \cdots$ $\mathrm{Ca}$ electrostatic interaction, as suggested by the electrostatic potential maps (see Figure 3). Indeed, the $\mathrm{C}=\mathrm{O} \cdots \mathrm{Ca}$ distance is in the 2.30-2.45 $\AA$ range (see Table 1), depending on the PPII side chain conformation (Figures 2 and 4 ).

The results in Table 1 for the D1 oriented polymer indicate that PRO3 puckering does not influence the adduct features $(R$, $\alpha$, and $\left.\mathrm{BE}^{* \mathrm{C}}\right)$. This is understandable, considering that PRO1 and $\mathrm{PRO} 2$ are in contact with the surface, while PRO3 points perpendicular to the surface. $\mathrm{PRO} 3$ puckering indirectly affects the $\mathrm{BE}^{\mathrm{C}}$ value through the deformation energy, $\delta E_{\mathrm{p}}$. For $\mathrm{D}$ puckering in $\mathrm{PRO} 3, \delta E_{\mathrm{P}}$ is smaller and so $\mathrm{BE}^{\mathrm{C}}$ is larger (see Table 1).
We computed the relative stabilities of the PPII as free polymer and when adsorbed on the HAP surface. The comparison reveals that HAP adhesion stabilizes conformers, which are inaccessible to the free polymer. The free UDD, UUD, and UUU conformers are $9.7 \mathrm{~kJ} \cdot \mathrm{mol}^{-1}, 17.8 \mathrm{~kJ} \cdot \mathrm{mol}^{-1}$, and $28.3 \mathrm{~kJ} \cdot \mathrm{mol}^{-1}$ less stable than the DDD (Table S2 of the Supporting Information, SI). On the HAP, the presence of $U$ puckered PROs in PRO1 or PRO2 positions increases the $\mathrm{BE}$ with the surface with respect to the DDD/HAP case. This effect is quantified to be $\sim 48 \mathrm{~kJ} \cdot \mathrm{mol}^{-1}$ at least, as revealed by the comparison of $\mathrm{BE}^{* \mathrm{C}}$ values for rows DDD and DUD/DUU of Table 1. This, in turn, shows that the UDD/HAP, UUD/HAP and UUU/HAP cases are more stable than DDD/HAP, at variance with respect to the free PPII polymer (vide supra).

To reduce the computational burden for the D2 alignment, we locked PRO3 in the D state, due to the low impact of PRO3 puckering on the PPII/HAP system as revealed by the analysis of all cases for the D1 alignment. Therefore, we ended up with only four distinct PPII conformers at the HAP surface. Data in Table 2 shows that the DDD conformer is much more tightly bound than for the D1 alignment (92.4 vs $\left.62.6 \mathrm{~kJ} \mathrm{~mol}^{-1}\right)$. We found that PRO1 puckering influences the characteristics of the PPII/HAP adduct more than the PRO2 puckering. For instance, the DDD/HAP adduct is as stable as the DUD/ $\mathrm{HAP}$ one. The largest $\mathrm{BE}^{\mathrm{C}}$ values are computed for the UDD/ $\mathrm{HAP}$ and UUD/HAP cases, in agreement with the results for the D1 alignment. Besides the already commented DDD case, all PPII conformers prefer to adsorb on HAP in the D1 alignment. For this reason, the dynamic behavior of PPII on HAP will be studied only for the D1 polymer alignment (vide infra).

The largest binding energy resulted for D1 oriented UDD/ $\mathrm{HAP}$ and $\mathrm{UUD} / \mathrm{HAP}$ cases $\left(\mathrm{BE}^{\mathrm{C}}\right.$ of $126.1 \mathrm{~kJ} \cdot \mathrm{mol}^{-1}$ and 126.3 $\mathrm{kJ} \cdot \mathrm{mol}^{-1}$, respectively). The origin of these values is due to electrostatic and London interactions acting in the same direction. The charge transfer between PPII and the HAP surface is, indeed, negligible: 0.02 and $0.001 e$ at the PBE-D2 and B3LYP-D2 level, respectively. The analysis of the various terms accounting for the final $\mathrm{BE}^{\mathrm{C}}$ (see eqs $1-6$ ) revealed that (i) the deformation terms are large for both the polymer and the surface due to the imposed matching between the two systems, and (ii) dispersion interactions account for about $50 \%$ of the final $\mathrm{BE}^{\mathrm{C}}$ values.

We studied the dependency of the binding energies on the role of exact exchange (B3LYP) and/or dispersion scheme $\left(\mathrm{D} 3^{\mathrm{ABC}}\right.$ ), proving the reliability of the PBE-D2 results (see Tables $S 6$ and S7). The PBE-D3 ${ }^{\mathrm{ABC}}$ BEs are larger by $\sim 40 \mathrm{~kJ}$. $\mathrm{mol}^{-1}$ compared to the PBE-D2 ones. This is due to the $\mathrm{C}_{6}$ term associated with the $\mathrm{Ca}$ atom which cannot be excluded in $\mathrm{D} 3^{\mathrm{ABC}}$ scheme, as coded in the CRYSTAL program, as we did for the D2 scheme (see Computational Methods). Nevertheless, calculations at PBE-D2 inclusive of the $\mathrm{C}_{6}$ term give $\mathrm{BEs}$ within a few $\mathrm{kJ} \cdot \mathrm{mol}^{-1}$ with respect to the PBE-D3 ${ }^{\mathrm{ABC}}$ values. Conversely, B3LYP-D $3^{\mathrm{ABC}} \mathrm{BEs}$ are always larger than PBE-D $3^{\mathrm{ABC}}$ ones by $33 \mathrm{~kJ} \cdot \mathrm{mol}^{-1}$, on average.

Figure 5 compares the relative stability, for the D1 alignment only, of the various conformers for three different situations: (i) the PPII as a free polymer; (ii) the PPII as a free polymer with the cell parameter fixed at that of the HAP unit cell; (iii) the PPII/HAP system. Comparison between case (i) and (ii) revealed that stretching the unit cell size of the free PPII polymer as imposed by the adsorption at the HAP surface (from 9.120 to $9.538 \AA$ ) causes an increase in the instability of 
Table 1. Binding Energy Components $\left(\mathrm{kJ} \cdot \mathrm{mol}^{-1}\right)($ Eqs $1-6$ in the Text) and Relevant Structural Data of the PPII/HAP System Relaxed at the PBE-D2/TZP Level ${ }^{a}$

\begin{tabular}{|c|c|c|c|c|c|c|c|c|c|}
\hline PPII (D1) & $\mathrm{BE}$ & $\mathrm{BE}^{b}$ & $\delta E_{\mathrm{S}}$ & $\delta E_{\mathrm{P}}$ & $\mathrm{BE}^{b \mathrm{C}}$ & $\mathrm{BE}^{\mathrm{C}}$ & \%BSSE & $\mathrm{R}$ & $\alpha$ \\
\hline DDD & 103.0 & 189.1 & 29.8 & 56.4 & 148.8 & 62.6 & 39.2 & 2.38 & 173 \\
\hline Disp & 39.0 & 46.1 & -0.1 & 7.1 & 46.1 & 39.0 & & & \\
\hline $\mathrm{DDU}(\mathrm{UDU})^{b}$ & 157.0 & 254.8 & 43.1 & 54.8 & 212.9 & 115.0 & 26.7 & 2.44 & 127 \\
\hline Disp & 58.6 & 61.9 & -0.7 & 4.0 & 61.9 & 58.6 & & & \\
\hline DUD & 144.4 & 220.6 & 20.5 & 55.7 & 186.5 & 110.4 & 23.6 & 2.33 & 150 \\
\hline Disp & 48.7 & 50.4 & -1.0 & 2.7 & 50.4 & 48.7 & & & \\
\hline UDD & 168.2 & 255.5 & 42.8 & 44.5 & 213.3 & 126.1 & 25.1 & 2.45 & 126 \\
\hline Disp & 60.4 & 61.9 & -0.8 & 2.3 & 61.9 & 60.4 & & & \\
\hline DUU & 136.5 & 220.8 & 20.7 & 63.7 & 186.7 & 102.3 & 25.0 & 2.32 & 151 \\
\hline Disp & 48.3 & 50.4 & -1.0 & 3.2 & 50.4 & 48.3 & & & \\
\hline UDU & 158.1 & 254.3 & 42.8 & 53.3 & 212.4 & 116.3 & 26.5 & 2.44 & 127 \\
\hline Disp & 59.6 & 61.8 & -0.7 & 2.9 & 61.8 & 59.6 & & & \\
\hline UUD & 164.8 & 239.0 & 36.7 & 37.5 & 200.5 & 126.3 & 23.4 & 2.42 & 131 \\
\hline Disp & 56.5 & 57.6 & -0.8 & 1.8 & 57.6 & 56.5 & & & \\
\hline UUU & 153.3 & 235.1 & 36.3 & 45.5 & 197.4 & 115.6 & 24.6 & 2.43 & 134 \\
\hline Disp & 55.5 & 56.6 & -0.6 & 1.8 & 56.6 & 55.5 & & & \\
\hline
\end{tabular}

${ }^{a}$ Results for the PPII D1 alignment (Figure 4). $R(\AA)$ and $\alpha$ (degree) are the distance between the oxygen atom of the C=O group and the Cal ion and the $\mathrm{C}=\mathrm{O} \cdots \mathrm{Cal}$ angle. ${ }^{b}$ During the geometry optimization, the PPII changes conformation from DDU/HAP to UDU/HAP.

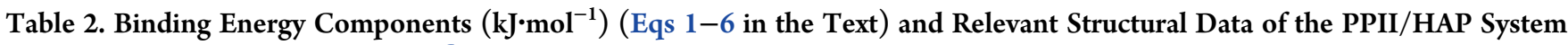
Relaxed at the PBE-D2/TZP Level ${ }^{a}$

\begin{tabular}{|c|c|c|c|c|c|c|c|c|c|}
\hline PPII (D2) & $\mathrm{BE}$ & $\mathrm{BE}^{*}$ & $\delta \mathrm{E}_{\mathrm{S}}$ & $\delta \mathrm{E}_{\mathrm{P}}$ & $\mathrm{BE}^{* \mathrm{C}}$ & $\mathrm{BE}^{\mathrm{C}}$ & \%BSSE & $\mathrm{R}$ & $\alpha$ \\
\hline DDD & 129.1 & 194.7 & 20.7 & 44.9 & 158.0 & 92.4 & 28.4 & 2.36 & 167 \\
\hline Disp & 37.0 & 47.9 & -0.2 & 11.1 & 47.9 & 37.0 & 0.0 & & \\
\hline UDD & 139.1 & 176.0 & 15.5 & 21.4 & 143.2 & 106.3 & 23.6 & 2.33 & 154 \\
\hline Disp & 42.3 & 44.0 & 0.3 & 1.4 & 44.0 & 42.3 & 0.0 & & \\
\hline DUD & 125.9 & 183.0 & 16.3 & 40.9 & 150.2 & 93.0 & 26.1 & 2.33 & 169 \\
\hline Disp & 41.8 & 44.0 & -0.3 & 2.5 & 44.0 & 41.8 & 0.0 & & \\
\hline UUD & 142.7 & 179.0 & 10.9 & 25.5 & 145.9 & 109.5 & 23.3 & 2.30 & 157 \\
\hline Disp & 43.4 & 45.6 & 0.1 & 2.1 & 45.6 & 43.4 & 0.0 & & \\
\hline
\end{tabular}

${ }^{a}$ Results for the PPII D2 alignment (Figure 4). $R(\AA)$ and $\alpha$ (degree) are the distance between the oxygen atom of the C=O group and the Ca1 ion and the $\mathrm{C}=\mathrm{O} \cdots \mathrm{Ca}$ angle.

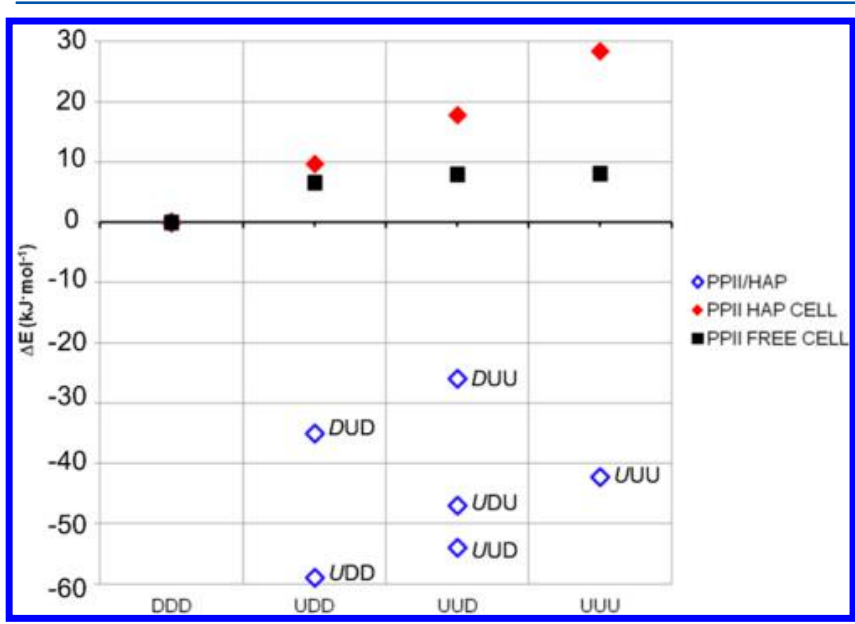

Figure 5. Relative stability with respect to the DDD conformer $\Delta E=$ $E_{\mathrm{XXX}}-E_{\mathrm{DDD}} ; \mathrm{X}$ is either $\mathrm{U}$ or $\mathrm{D}$. Cases are the free PPII polymer fully relaxed (filled square) (see Table S1), the free PPII polymer relaxed with cell length fixed to the HAP $b$ cell parameter (filled red diamond), and the PPII/HAP system (empty blue diamond) (see Table S2) in the D1 alignment.

the different conformations. Also, while the UDD, UUD, and UUU relative stability with respect to DDD are very close to each other, they dramatically differ from each other upon stretching the PPII unit cell. Indeed, the UUU conformer is 3 times more unstable when stretched than the free conformer. This is due to the intrinsic contraction of the polymer unit cell when switching from the DDD to the others conformers (see Table S1). Figure 5 also highlights how the adsorption of PPII at the HAP surface brings about a dramatic change of the relative stability compared to the free (stretched) polymer. Indeed, the UDD conformation becomes the most stable one at the HAP surface by about $60 \mathrm{~kJ} \mathrm{~mol}^{-1}$. Energy differences reveals that the population of all other conformations, except the UUD case, are almost negligible.

We studied possible correlations between the energetic data of Table 1, responsible for the stabilities shown in Figure 5, and the geometrical details of both the PPII polymer and the HAP surface. We only find a good correlation between the displacements of the $z$ coordinate of the $\mathrm{Cal}$ ion from the equilibrium position of the free HAP with the deformation energy $\delta \mathrm{E}_{\mathrm{S}}$ of the HAP surface (see Figure 6, left). This is in agreement with the localized character of the PPII/HAP interaction, specifically due to the $\mathrm{C}=\mathrm{O} \cdots \mathrm{Ca} 1$ bond. Figure 6 right, reveals that the deformation energy $\Delta E_{\mathrm{P}}$ of the polymer (see eq 7) only roughly correlates with the variation of the PPII torsional angles, dictating the polymer conformation.

Dynamics of PPII Polymer Free and Adsorbed at HAP. In a previous work from some of us, ${ }^{27}$ we compute the energy 

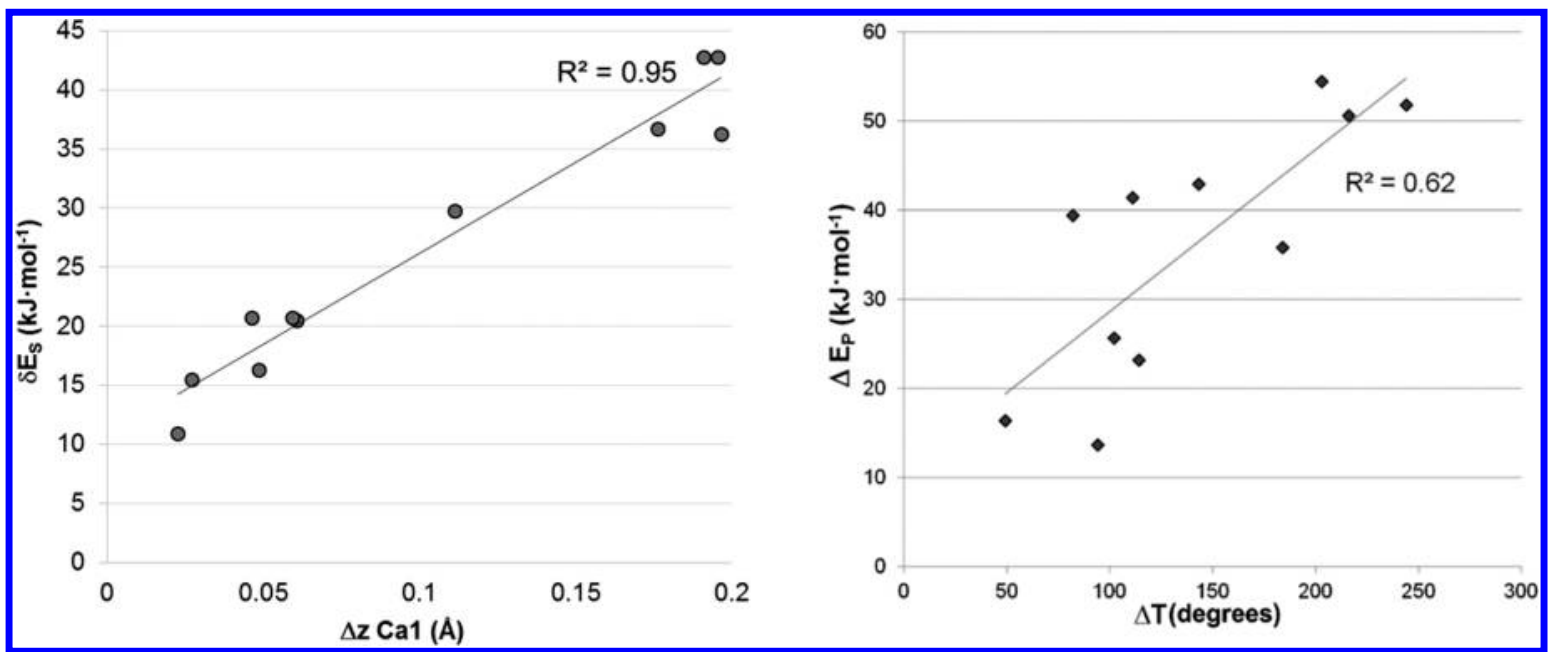

Figure 6. Left: surface deformation energy $\left(\delta E_{\mathrm{S}}\right)$ versus the displacement of the $\mathrm{z}$ coordinate of the most exposed Cal ion $(\Delta \mathrm{z})$ with respect to the clean surface. All PPII/HAP cases of Table 1 and Table 2 are included. Right: polymer deformation energy $\Delta E_{\mathrm{P}}$ versus $\Delta T$. $\Delta E_{\mathrm{P}}$ is the energy difference between the relaxed free PPII with cell length fixed to the $b$ HAP cell vector and the PPII adsorbed onto the HAP surface (see eq 7$)$. $\Delta T=$ $\left|\phi^{\text {ads }}-\phi^{\mathrm{r}}\right|+\left|\omega^{\mathrm{ads}}-\omega^{\mathrm{r}}\right|+\left|\psi^{\mathrm{ads}}-\psi^{\mathrm{r}}\right|$, where $\phi^{\mathrm{ads}}, \omega^{\mathrm{ads}}$ and $\psi^{\mathrm{ads}}$ are the backbone dihedral angles of the polymer adsorbed on to the HAP surface and $\phi^{\mathrm{r}}$, $\omega^{\mathrm{r}}$, and $\psi^{\mathrm{r}}$ those of the relaxed polymer with the cell length fixed at the HAP unit cell $b$ parameter.

barrier of a $\mathrm{D} \rightarrow \mathrm{U}$ ring flipping for the PPII free polymer to be only $8 \mathrm{~kJ} \cdot \mathrm{mol}^{-1}$, with a half-life time of $2.8 \mathrm{ps}$ at $\mathrm{RT}^{27}$ Therefore, it is important to study the structural evolution in time, if any, of the PPII/HAP system. To that purpose, we run AIMD calculations (see Computational Method section) for PPII, both as a free polymer and when adsorbed on the HAP surface. The advantage of dynamic simulations is including thermal and entropic effects, which are neglected in the static approaches. To start with, we set the free PPII polymer structure in the DDD state and the adsorbed one in the DDD/ HAP conformation. The DDD state is the most stable one from static calculations. We only considered the PPII on the HAP surface in its most stable D1 configuration.

During the dynamic of the free PPII polymer, two of the four available conformational states, DDD and UDD, are visited. A statistical analysis of the trajectories for the whole production time revealed that DDD and UDD minima are populated for $92 \%$ and $8 \%$ of the simulation time, respectively (see Figure 7 ). This result is in good agreement with the prediction based on static calculations (see Figure 5). Indeed, from a Boltzmann analysis of the static PBE-D2/TZP relative stabilities of PPII at $T=300 \mathrm{~K}$ the occupancy of the DDD, UDD, UUD, and UUU conformers is $87 \%, 6 \%, 4 \%$ and $3 \%$, respectively. Dynamical simulations can give an estimation of the ring and main chain flexibility. We monitored the range of fluctuation of the PPII backbone dihedral angles during the dynamic, represented by their standard deviation (SD) with respect to the average value. We calculated comparable flexibility results for all the torsion angles. Indeed $\phi, \psi$, and $\omega$ gave SD of $10^{\circ}, 9^{\circ}$, and $8^{\circ}$, respectively, averaged on all the PROs. Static and dynamic geometries on the $\phi$ angle are in perfect agreement: $-72^{\circ}$ and $-72^{\circ} \pm 10^{\circ}$, respectively. Conversely, for $\psi$ and $\omega$, the static/ dynamic deviation is at least of $8^{\circ}$.

When the PPII polymer interacts with the HAP surface, the conformation evolves from the DDD/HAP to the UDD/HAP state (see Figure 7). The $\mathrm{D} \rightarrow \mathrm{U}$ ring flipping occurs immediately during the equilibration time (first $2.4 \mathrm{ps}$ ). Interestingly, the dynamic of the event is in agreement with the estimated half-life time, $\tau_{1 / 2}$, for a DDD $\rightarrow$ UDD ring flip for the free polymer $\left(\tau_{1 / 2}=2.8 \mathrm{ps}\right)$, as previously computed in ref
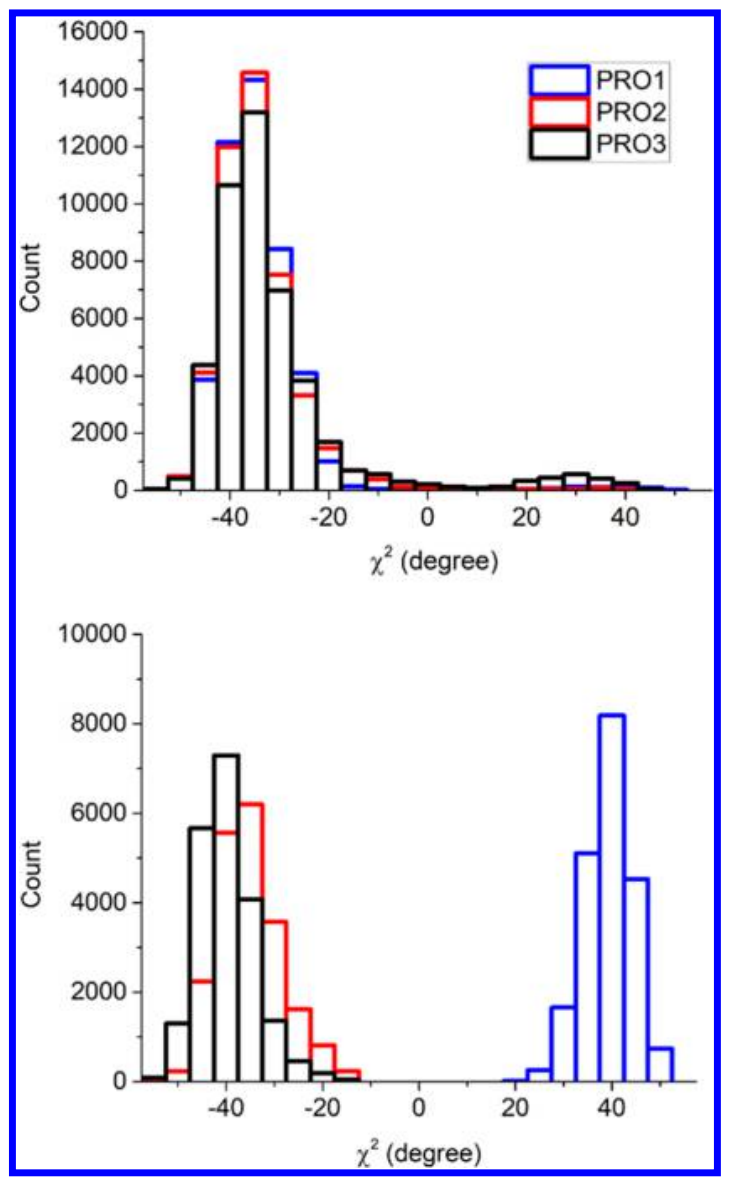

Figure 7. Frequency of occurrence of $\chi^{2}$ (ring) torsional angle for PRO1, PRO2, and PRO3 during the dynamics for the free PPII polymer (top graph) and when adsorbed at the HAP surface (bottom graph).

27. After the initial flip, the UDD/HAP state remains stable for the whole simulation, confirming the result of the static simulations. As already pointed out, the static simulations indicate the UDD/HAP and UUD/HAP as the lowest energy 


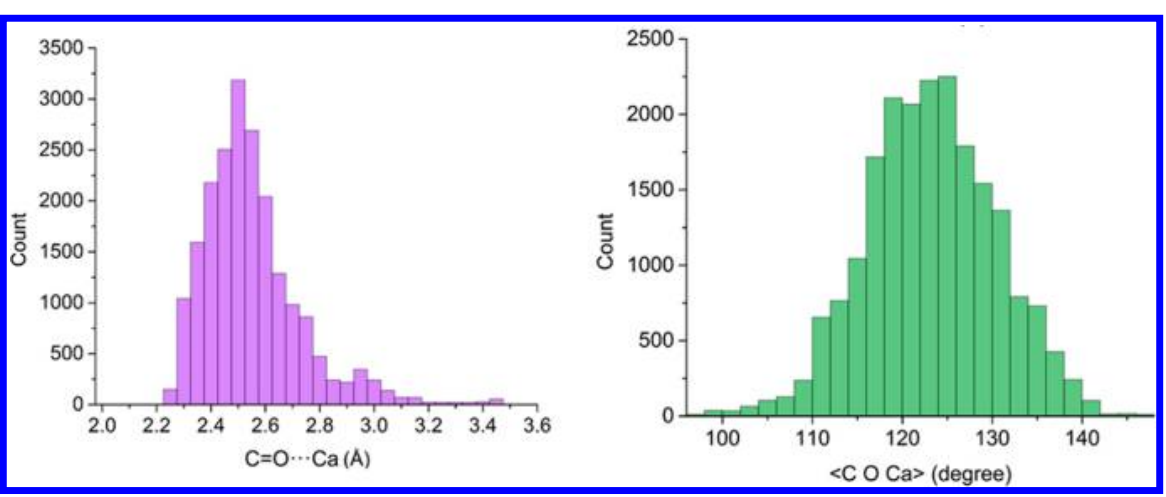

Figure 8. PRO1-Ca frequency count for the $\mathrm{C}=\mathrm{O} \cdots \mathrm{Cal}$ distance (left) and angle (right) during the PPII/HAP dynamic calculation $(\mathrm{C}=\mathrm{O} \cdots \mathrm{Ca}$ angle $\mathrm{ME} \pm \mathrm{SD}$ of $123.2 \pm 7.2)$.

states for the PPII/HAP system. Therefore, we may expect the dynamic simulation to evolve from UDD/HAP to UUD/HAP providing a long enough production time. This is not guaranteed, as we have no information on the energy barrier for the UDD/HAP $\rightarrow U U D / H A P$ reaction. This point can be efficiently addressed adopting meta-dynamics methods or by computing, through static calculations, the potential energy surface of the PROs ring. Unfortunately, these computational procedures are outside our computational facilities. Nevertheless, the equilibrated structures can serve as a good starting point for future, more sophisticated, calculations.

During the dynamics, the system is anchored onto the surface stably in one state, as shown in Figure 8. Indeed, the $\mathrm{C}=\mathrm{O} \cdots \mathrm{Ca} 1$ distances indicate an almost pure one-modal distribution. Moreover, the angle between the $\mathrm{C}=\mathrm{O} \cdots \mathrm{Ca} 1$ oscillates around the average $120^{\circ}$, in agreement with the oxygen $\mathrm{sp}^{2}$ hybridization dictating the final geometry (Figure 8).

As expected, the surface adsorption induces a geometrical distortion of the polymer torsion bonds to maximize the contact with the HAP surface, which can be summarized as follows:

(i) For the backbone dihedrals, we computed the Ramachandran plot of the free and adsorbed PPII polymer, as shown in Figure 9. It is worth noting that PRO3, which is the residue least involved with the HAP surface, has the largest deviation on the $(\phi, \psi)$ values with respect to the free PPII system. Indeed, the PRO1 and PRO2 regions of existence largely overlap with those of the free PPII one (yellow area on the graph), including also the ideal value for PPII conformation (black cross on the graph).

(ii) For the side chain puckering, Figure 7 clearly shows the evolution of the $\chi^{2}$ torsional angle from a one-modal distribution type for the free PPII polymer to a bimodal one for PPII at HAP.

(iii) For the bond mobility, we computed bonds and side chain ring stiffer on HAP rather than in gas phase (see Figure S3 and S4). The larger the interaction, the larger the effect. Indeed, the most affected amino-acid is PRO1, which is in direct contact with the surface. The average $\mathrm{SD}$ is reduced to $2.2^{\circ}, 1.5^{\circ}$, and $1.6^{\circ}$ in the adsorption process for PRO1, PRO2, and PRO3, respectively. Due to the mutual connection between main and side chain pucker, the main chain increased stiffness also affects the side chain.

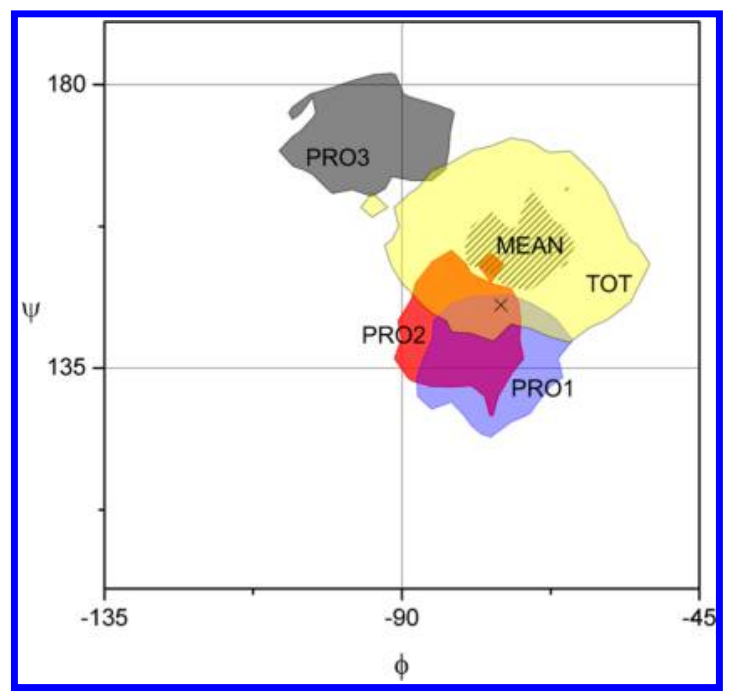

Figure 9. Ramachandran plot for the PPII polymer dynamic both free and adsorbed on HAP. Each value of $(\phi, \psi)$ is gathered into square bins with edge of $3^{\circ}$. The colored surface highlights the center of the most filled bins (with $2 / 3$ of the hits of the most filled bin at least) Color code for the PPII/HAP dynamic: PRO1 in blue, PRO2 in red, PRO3 in dark gray. For the dynamic of the free PPII, we report the $(\phi, \psi)$ region visited by all PROs (TOT, in yellow) as well as the mean values (as black lines). The black cross represents the most probable $(\phi, \psi)$ values for the free PPII, $\left(-75^{\circ}, 145^{\circ}\right) .57$

It is important to point out that the agreement between UDD/HAP geometry computed by static and dynamical simulations is remarkable. The dynamical average values of the adsorbed PPII at HAP differ from the static result by less than $1^{\circ}$ on average (for $\phi, \psi, \omega$ and $\chi^{2}$, see Table S3 and Figure $\mathrm{S} 3)$. Furthermore, the $\mathrm{C}=\mathrm{O} \cdots \mathrm{Cal}$ distance and angle from static simulations fit well within the computed dynamic oscillations (see Table 1 and Figures 8 and S6).

\section{CONCLUSIONS}

In this work, we have defined and investigated a simplified periodic model of the collagen polymer envisaging a singlestrand of poly-L-proline type II polymer (PPII). We studied the static and dynamic behavior of the conformational features for the dry PPII, either free or adsorbed on the P-rich (010) surface of hydroxyapatite (HAP), one of the extended surfaces of the key inorganic component of bones. We adopted density functional methods based on both gradient corrected (PBE) and hybrid (B3LYP) functionals, augmented with the Grimme's 
empirical correction (D2 and D3) to account for the dispersion interactions. We have focused on the role of the proline (PRO) side chain conformations in dictating the features of the PPII and how they are affected by the interaction with the HAP surface. To that purpose, we run static and dynamic calculations at the PBE-D2 level of theory to assess the relative population of each possible conformers for the free and adsorbed PPII polymer. The main findings of our work are

(i) The PPII adsorbs on hydroxyapatite surface with positive binding energies. The process is guided by a moderate electrostatic interaction between a PRO carbonyl group and the most exposed $\mathrm{Ca}$ ion of the HAP surface. The $\mathrm{C}=\mathrm{O} \cdots$ Ca distance covers the $2.30-2.45 \AA$ range, while the adsorption binding energy spans the $63-126 \mathrm{~kJ}$. $\mathrm{mol}^{-1}$ range. The values fluctuation depends on the puckering state of the PROs and the polymer alignment with respect to the surface unit cell axes. The charge transfer occurring during the adsorption process is small at the PBE-D2 level and negligible at the B3LYP-D2 level. In the adhesion process, the polymer loses its helicity, so that the adsorbed PPII has two PRO residues parallel and one perpendicular to the surface. The puckering of the PROs has a leading role in dictating the stability of PPII at the surface. In addition to that, the alignment (D1 or D2) of the polymer toward the surface affects the adduct stability by a different sampling of the surface electrostatic potential.

(ii) The hydroxyapatite surface is modified by the interaction with the PPII polymer mainly through the lifting of the most exposed Ca ion directly interacting with the PRO's $\mathrm{C}=\mathrm{O}$ group. The much more flexible PPII polymer strongly deforms to stay in contact with the surface by altering all the internal degrees of freedom.

(iii) The binding energy comprises a repulsive and an attractive component. The former arises from the geometrical deformation of both polymer and surface as adsorption consequence. The latter derives from the electrostatic and dispersion interactions. Overall the binding energy is always attractive, and dispersion interaction contributes to more than half of its final value. Therefore, it must be included in any kind of computer simulation on this type of system. We also showed dispersion to be almost insensitive to the adopted computational scheme, e.g., $\mathrm{D} 2$ or $\mathrm{D} 3^{\mathrm{ABC}}$. The hybrid B3LYP functional computes larger BEs $(40 \mathrm{~kJ}$. $\mathrm{mol}^{-1}$ on average) than PBE only, without affecting the relative stability between free ${ }^{27}$ and adsorbed PPII conformations. The surface deformation energy correlates with the height of the most exposed $\mathrm{Ca}$ ion at the hydroxyapatite surface as caused by the PPII adsorption. Conversely, the polymer deformation energy correlates only broadly with the geometrical distortion of the torsional degrees of freedom of the adsorbed PPII polymer.

(iv) The PPII interaction with the hydroxyapatite surface is such that PPII conformers that are usually unstable for the free PPII polymer become stabilized at the surface. As free polymer, the PPII exists mainly in the DDD conformation. At room temperature, static simulations indicate an occupancy of $87 \%, 6 \%, 4 \%$, and $3 \%$ for DDD, UDD, UUD, and UUU conformational states. Inclusion of thermic and entropic terms by dynamic simulations does indeed confirm the static population distribution. During the dynamic, the PPII visits the DDD and UDD states for the $92 \%$ and $8 \%$ of the time, respectively. Conversely, on the HAP surface, static simulations indicate that the UDD/HAP and UUD/HAP states are the most stable ones, regardless of the polymer alignment toward the hydroxyapatite unit cell axes. During the dynamic, only the UDD/HAP conformation is visited by the polymer, which is stable for the whole simulation time. The interaction between U-PROs and the HAP surface takes place in bone through HYP residues, the side chain of which is always $U$ puckered. Moreover, $\mathrm{HYP} \mathrm{C}=\mathrm{O}$ group do not usually participate to the interstrand $\mathrm{H}$ bond in COLs, which is free to interact with the HAP surface. This may indicate a relevant role of the HYP residues in the COL/HAP interface in bones.

Despite the simplicity of the adopted models for the collagen polymer, these results may be useful for further refinement. The simplicity of the present model allows studying how solvation water will affect the relative stability of different PPII conformations, either free and when adsorbed at the HAP surface. Furthermore, in our laboratory, we are already testing a full triple-helix model of collagen by exploiting the internal helix symmetry to reduce the computational burden. In perspective, we will tune the above-mentioned collagen models as a compromise between structural complexity and computational cost to investigate the hybrid COL/HAP interface.

\section{ASSOCIATED CONTENT}

\section{S Supporting Information}

The Supporting Information is available free of charge on the ACS Publications website at DOI: 10.1021/acs.jpcc.7b10013.

Structures of the PPII free and adsorbed polymers (Tables S1-S5), adsorption energies of PPII on HAP surface (Tables S6, S7), AIMD energetic (Figures S1, S2), and structural features of the PPII both free and adsorbed (Figures S3-S6) (PDF)

\section{AUTHOR INFORMATION}

\section{Corresponding Author}

*E-mail: piero.ugliengo@unito.it.

ORCID

Marta Corno: 0000-0001-7248-2705

Dominique Costa: 0000-0002-3781-9867

Piero Ugliengo: 0000-0001-8886-9832

\section{Present Address}

${ }^{\S}$ Dipartimento di Scienze e Innovazione Tecnologica, Università del Piemonte Orientale, Viale Teresa Michel 11, 15121 Alessandria, Italy

\section{Notes}

The authors declare no competing financial interest.

\section{ACKNOWLEDGMENTS}

P.U. acknowledges the generous allowance of computing time from ISCRA Project BONECOLL, Project Code HP10BPE1K6, run at CINECA supercomputing centre.

\section{REFERENCES}

(1) Fratzl, P. Collagen. Structure and Mechanics; Fratzl, P., Ed.; Springer Berlin/Heidelberg: Postdam, Germany, 2008. 
(2) Stock, S. R. The Mineral-Collagen Interface in Bone. Calcif. Tissue Int. 2015, 97, 262.

(3) Ramshaw, J. A. M.; Shah, N. K.; Brodsky, B. Gly-X-Y Tripeptide Frequencies in Collagen: A Context for Host-Guest Triple-Helical Peptides. L. Struct. Biol. 1998, 122 (1-2), 86-91.

(4) Shoulders, M. D.; Raines, R. T. Collagen Structure and Stability. Annu. Rev. Biochem. 2009, 78, 929-958.

(5) Orgel, J. P. R. O.; Irving, T. C.; Miller, A.; Wess, T. J. Microfibrillar Structure of Type I Collagen in Situ. Proc. Natl. Acad. Sci. U. S. A. 2006, 103 (24), 9001.

(6) Hodge, A. J.; Petruska, J. A. Recent Studies with the Electron Microscope on Ordered Aggregates of the Tropocollagen Molecule. In Aspects of Protein Structure; Ramachandran, G. N., Ed.; Academic Press: New York, 1963; pp 289-300.

(7) Landis, W. J.; Song, M. J.; Leith, A.; McEwen, L.; McEwen, B. F. Mineral and Organic Matrix Interaction in Normally Calcifying Tendon Visualized in Three Dimensions by High-Voltage Electron Microscopic Tomography and Graphic Image Reconstruction. I. Struct. Biol. 1993, 110, 39-54.

(8) Hassenkam, T.; Fantner, G. E.; Cutroni, J. A.; Weaver, J. C.; Morse, D. E.; Hansma, P. K. High-Resolution AFM Imaging of Intact and Fractured Trabecular Bone. Bone 2004, 35 (1), 4-10.

(9) Fratzl, P.; Groschner, M.; Vogl, G.; Plenk, H.; Eschberger, J.; Fratzl-Zelman, N.; Koller, K.; Klaushofer, K. Mineral Crystals in Calcified Tissues: A Comparative Study by SAXS. I. Bone Miner. Res. 1992, 7 (3), 329-334.

(10) Handschin, R. G.; Stern, W. B. X-Ray Diffraction Studies on the Lattice Perfection of Human Bone Apatite (Crista Iliaca). Bone 1995, $16,355 S-363 S$.

(11) Bar-On, B.; Wagner, H. D. Structural Motifs and Elastic Properties of Hierarchical Biological Tissues - A Review. $\underline{\text { I. Struct. Biol. }}$ 2013, 183 (2), 149-164.

(12) Nair, A. K.; Gautieri, A.; Chang, S.-W.; Buehler, M. J. Molecular Mechanics of Mineralized Collagen Fibrils in Bone. Nat. Commun. 2013, 4, 1724.

(13) Domene, C.; Jorgensen, C.; Abbasi, S. A Perspective on Structural and Computational Work on Collagen. Phvs. Chem. Chem. Phvs. 2016, 18, 24802.

(14) Almora-Barrios, N.; De Leeuw, N. Modelling the Interaction of a Hyp-Pro-Gly Peptide with Hydroxyapatite Surfaces in Aqueous Environment. CrystEngComm 2010, 12, 960-967.

(15) Tao, J.; Battle, K. C.; Pan, H.; Salter, E. A.; Chien, Y.-C.; Wierzbicki, A.; De Yoreo, J. J. Energetic Basis for the Molecular-Scale Organization of Bone. Proc. Natl. Acad. Sci. U. S. A. 2015, 112 (2), 326-331.

(16) Qin, Z.; Gautieri, A.; Nair, A. K.; Inbar, H.; Buehler, M. J. Thickness of Hydroxyapatite Nanocrystal Controls Mechanical Properties of the Collagen-Hydroxyapatite Interface. Langmuir 2012, 28 (4), 1982-1992.

(17) Almora-Barrios, N.; De Leeuw, N. H. A Density Functional Theory Study of the Interaction of Collagen Peptides with Hydroxyapatite Surfaces. Langmuir 2010, 26 (18), 14535-14542.

(18) Delle Piane, M.; Corno, M.; Orlando, R.; Dovesi, R.; Ugliengo, P. Elucidating the Fundamental Forces in Protein Crystal Formation: The Case of Crambin. Chem. Sci. 2016, 7 (2), 1496-1507.

(19) Orlando, R.; Delle Piane, M.; Bush, I. J.; Ugliengo, P.; Ferrabone, M.; Dovesi, R. A New Massively Parallel Version of CRYSTAL for Large Systems on High Performance Computing Architectures. I. Comput. Chem. 2012, 33 (28), 2276-2284.

(20) Hutter, J.; Iannuzzi, M.; Schiffmann, F.; Vandevondele, J. CP2K: Atomistic Simulations of Condensed Matter Systems. Wilev Interdiscip. Rev. Comput. Mol. Sci. 2014, 4 (1), 15-25.

(21) Corno, M.; Rimola, A.; Bolis, V.; Ugliengo, P. Hydroxyapatite as a Key Biomaterial: Quantum-Mechanical Simulation of Its Surfaces in Interaction with Biomolecules. Phys. Chem. Chem. Phvs. 2010, 12 (24), 6309.

(22) Corno, M.; Busco, C.; Bolis, V.; Tosoni, S.; Ugliengo, P. Water Adsorption on the Stoichiometric (001) and (010) Surfaces of
Hydroxyapatite: A Periodic B3LYP Study. Lanomuir 2009, 25 (4), 2188-2198.

(23) Jimenez-Izal, E.; Chiatti, F.; Corno, M.; Rimola, A.; Ugliengo, P. Glycine Adsorption at Nonstoichiometric (010) Hydroxyapatite Surfaces: A B3LYP Study. I. Phys. Chem. C 2012, 116 (27), 1456114567.

(24) Rimola, A.; Corno, M.; Zicovich-Wilson, C. M.; Ugliengo, P. Ab Initio Modeling of Protein/biomaterial Interactions: Glycine Adsorption at Hydroxyapatite Surfaces. I. Am. Chem. Soc. 2008, 130, 1618116183.

(25) Rimola, A.; Corno, M.; Garza, J.; Ugliengo, P. Ab Initio Modelling of Protein-Biomaterial Interactions: Influence of Amino Acid Polar Side Chains on Adsorption at Hydroxyapatite Surfaces. Philos. Trans. R. Soc., A 2012, 370 (1963), 1478-1498.

(26) Rimola, A.; Aschi, M.; Orlando, R.; Ugliengo, P. Does Adsorption at Hydroxyapatite Surfaces Induce Peptide Folding? Insights from Large-Scale B3LYP Calculations. I. Am. Chem. Soc. 2012, 134 (26), 10899-10910.

(27) Cutini, M.; Corno, M.; Ugliengo, P. Method Dependence of Proline Ring Flexibility in the Poly-L-Proline Type II Polymer. $L$ Chem. Theory Comput. 2017, 13, 370-379.

(28) Ying Chow, W.; Bihan, D.; Forman, C. J.; Slatter, D. A.; Reid, D. G.; Wales, D. J.; Farndale, R. W.; Duer, M. J. Hydroxyproline Ring Pucker Causes Frustration of Helix Parameters in the Collagen Triple Helix. Sci. Rep. 2015, 5, 12556.

(29) Sure, R.; Grimme, S. Corrected Small Basis Set Hartree-Fock Method for Large Systems. I. Comput. Chem. 2013, 34, 1672-1685.

(30) Dovesi, R.; Orlando, R.; Erba, A.; Zicovich-Wilson, C. M.; Civalleri, B.; Casassa, S.; Maschio, L.; Ferrabone, M.; De La Pierre, M.; D'Arco, P.; et al. CRYSTAL14: A Program for the Ab Initio Investigation of Crystalline Solids. Int. I. Quantum Chem. 2014, 114 (19), 1287-1317.

(31) Perdew, J. P.; Burke, K.; Ernzerhof, M. Generalized Gradient Approximation Made Simple. Phvs. Rev. Lett. 1996, 77 (18), 38653868.

(32) Grimme, S. Semiempirical GGA-Type Density Functional Constructed with a Long-Range Dispersion Correction. I. Comput. Chem. 2006, 27 (15), 1787-1799.

(33) Broyden, C. G. The Convergence of a Class of Double-Rank Minimization Algorithms 1. General Considerations. IMA I. Appl. Math. 1970, 6 (1), 76-90.

(34) Fletcher, R. A. New Approach to Variable Metric Algorithms. Comput. I. 1970, 13, 317-322.

(35) Shanno, D. F.; Kettler, P. C. Optimal Conditioning of QuasiNewton Methods. Math. Comput. 1970, 24 (111), 657-664.

(36) Monkhorst, H. J.; Pack, J. D. Special Points for Brillouin-Zone Integration. Phvs. Rev. B 1976, 13, 5188-5192.

(37) Dovesi, R.; Saunders, V. R.; Roetti, C.; Orlando, R.; ZicovichWilson, C. M.; Pascale, F.; Civalleri, B.; Doll, K.; Harrison, N. M.; Bush, I. J.; et al. CRYSTAL14, User's Manual; Università di Torino: Torino, Italy, 2014

(38) Prencipe, M.; Pascale, F.; Zicovich-Wilson, C. M.; Saunders, V. R; Orlando, R; Dovesi, R. The Vibrational Spectra of Calcite (CaCO3): An Ab Inito Quantum-Mechanical Calculation. Phvs. Chem. Miner. 2004, 31, 559-564.

(39) Pulay, P. Improved SCF Convergence Acceleration. I. Comput. Chem. 1982, 3 (4), 556-560.

(40) Schäfer, A.; Horn, H.; Ahlrichs, R. Fully Optimized Contracted Gaussian Basis Sets for Atoms Li to Kr. I. Chem. Phvs. 1992, 97, 2571.

(41) Corno, M.; Busco, C.; Civalleri, B.; Ugliengo, P. Periodic Ab Initio Study of Structural and Vibrational Features of Hexagonal Hydroxyapatite Ca10(PO4)6(OH)2. Phvs. Chem. Chem. Phvs. 2006, 8 , 2464-2472.

(42) Becke, A. D. Density-Functional Exchange-Energy Approximation with Correct Asymptotic Behavior. Phvs. Rev. A: At., Mol., Opt. Phvs. 1988, 38 (6), 3098-3100.

(43) Becke, A. D. Density-Functional Thermochemistry. III. The Role of Exact Exchange. I. Chem. Phys. 1993, 98 (7), 5648-5652. 
(44) Lee, C.; Yang, W.; Parr, R. G. Development of the Colle-Salvetti Correlation-Energy Formula into a Functional of the Electron Density. Phvs. Rev. B: Condens. Matter Mater. Phvs. 1988, 37 (2), 785-789.

(45) Grimme, S.; Antony, J.; Ehrlich, S.; Krieg, H. A Consistent and Accurate Ab Initio Parametrization of Density Functional Dispersion Correction (DFT-D) for the 94 Elements H-Pu. J. Chem. Phys. 2010, 132, 154104.

(46) Muto, Y. The Force Between Nonpolar Molecules. Proc. Phys. Math. Soc. Japan 1944, 17, 629.

(47) Axilrod, B. M.; Teller, E. Interaction of the van Der Waals Type Between Three Atoms. L. Chem. Phys. 1943, 11, 299-300.

(48) Kohlmeyer, A.; Mundy, C. J.; Mohamed, F.; Schiffmann, F.; Tabacchi, G.; Forbert, H.; Kuo, W.; Hutter, J.; Krack, M.; Iannuzzi, M.; et al. CP2K; CP2K Developers Group, 2004.

(49) Vandevondele, J.; Krack, M.; Mohamed, F.; Parrinello, M.; Chassaing, T.; Hutter, J. Quickstep: Fast and Accurate Density Functional Calculations Using a Mixed Gaussian and Plane Waves Approach. Comput. Phvs. Commun. 2005, 167 (2), 103-128.

(50) Hartwigsen, C.; Goedecker, S.; Hutter, J. Relativistic Separable Dual-Space Gaussian Pseudopotentials from H to Rn. Phvs. Rev. B: Condens. Matter Mater. Phvs. 1998, 58 (7), 3641-3662.

(51) Nosé, S. A Unified Formulation of the Constant Temperature Molecular Dynamics Methods. I. Chem. Phys. 1984, 81, 511.

(52) Hoover, W. G. Canonical Dynamics: Equilibrium Phase-Space Distributions. Phvs. Rev. A: At., Mol., Opt. Phvs. 1985, 31 (3), 16951697.

(53) Ugliengo, P.; Viterbo, D.; Chiari, G. MOLDRAW: Molecular Graphics on a Personal Computer. Z. Kristallogr. - Cryst. Mater. 1993, 207, 9-23.

(54) Humphrey, W.; Dalke, A.; Schulten, K. VMD: Visual Molecular Dynamics. I. Mol. Graphics 1996, 14 (1), 33-38.

(55) Momma, K.; Izumi, F. VESTA 3 for Three-Dimensional Visualization of Crystal, Volumetric and Morphology Data. I. Appl. Crustallogr. 2011, 44 (6), 1272-1276.

(56) Boys, S. F.; Bernardi, F. The Calculation of Small Molecular Interactions by the Differences of Separate Total Energies. Some Procedures with Reduced Errors. Mol. Phys. 1970, 19, 553-566.

(57) Adzhubei, A. A.; Sternberg, M. J. E.; Makarov, A. A. PolyprolineII Helix in Proteins: Structure and Function. L.Mol. Biol. 2013, 425, $2100-2132$.

\section{NOTE ADDED AFTER ASAP PUBLICATION}

This paper was published January 5, 2018 with an incorrect address listed for Dominique Costa. This has been corrected and the revised version was re-posted November 21, 2018. 